\title{
Posttraumatic Psychological Symptoms are Associated with Reduced Inhibitory Control, not General Executive Dysfunction
}

\section{Citation}

DeGutis, Joseph, Michael Esterman, Bay McCulloch, Andrew Rosenblatt, William Milberg, and Regina McGlinchey. 2015. "Posttraumatic Psychological Symptoms Are Associated with Reduced Inhibitory Control, Not General Executive Dysfunction." Journal of the International Neuropsychological Society 21 (5): 342-52. doi:10.1017/S1355617715000235.

\section{Permanent link}

http://nrs.harvard.edu/urn-3:HUL.InstRepos:41542971

\section{Terms of Use}

This article was downloaded from Harvard University's DASH repository, and is made available under the terms and conditions applicable to Other Posted Material, as set forth at http:// nrs.harvard.edu/urn-3:HUL.InstRepos:dash.current.terms-of-use\#LAA

\section{Share Your Story}

The Harvard community has made this article openly available.

Please share how this access benefits you. Submit a story.

\section{Accessibility}




\title{
Posttraumatic Psychological Symptoms are Associated with Reduced Inhibitory Control, not General Executive Dysfunction
}

\author{
Joseph DeGutis, ${ }^{1,2}$ Michael Esterman, ${ }^{1,3}$ Bay McCulloch, ${ }^{1}$ Andrew Rosenblatt, ${ }^{4}$ William Milberg, ${ }^{1,5,6}$ AND \\ Regina McGlinchey ${ }^{1,5,6}$ \\ ${ }^{1}$ Translational Research Center for TBI and Stress Disorders (TRACTS), VA RR\&D TBI Center of Excellence, VA Boston Healthcare System, \\ Boston, Massachusetts \\ ${ }^{2}$ Department of Medicine, Harvard Medical School, Boston, Massachusetts \\ ${ }^{3}$ Department of Psychiatry, Boston University School of Medicine, Boston, Massachusetts \\ ${ }^{4}$ Department of Psychology, University of Houston, Houston, Texas \\ ${ }^{5}$ Geriatric Research Education and Clinical Center (GRECC), Boston Division VA Healthcare System, Boston, Massachusetts \\ ${ }^{6}$ Department of Psychiatry, Harvard Medical School, Boston, Massachusetts
}

(ReCEIVED January 15, 2014; Final Revision March 8, 2015; AcCEPTED March 15, 2015)

\begin{abstract}
Although there is mounting evidence that greater PTSD symptoms are associated with reduced executive functioning, it is not fully understood whether this association is more global or specific to certain executive function subdomains, such as inhibitory control. We investigated the generality of the association between PTSD symptoms and executive function by administering a broad battery of sensitive executive functioning tasks to a cohort of returning Operation Enduring Freedom/Operation Iraqi Freedom Veterans with varying PTSD symptoms. Only tasks related to inhibitory control explained significant variance in PTSD symptoms as well as symptoms of depression, while measures of working memory, measures of switching, and measures simultaneously assessing multiple executive function subdomains did not. Notably, the two inhibitory control measures that showed the highest correlation with PTSD and depressive symptoms, measures of response inhibition and distractor suppression, explained independent variance. These findings suggest that greater posttraumatic psychological symptoms are not associated with a general decline in executive functioning but rather are more specifically related to stopping automatic responses and resisting internal and external distractions. (JINS, 2015, 21, 1-11)
\end{abstract}

Keywords: Response inhibition, Distractor suppression, Posttraumatic stress disorder, Trauma, Depression, OEF/OIF veterans

\section{INTRODUCTION}

Posttraumatic stress disorder (PTSD) is a disabling clinical syndrome that threatens social and vocational functioning (Kessler et al., 2005). PTSD affects 6.8\% of American adults (Kessler et al., 2005) and is even more prevalent after military deployment $[13.8 \%$ of Operation Enduring Freedom/ Operation Iraqi Freedom (OEF/OIF) Veterans, Tanielian and Jaycox, 2008]. Studies suggest that poor outcomes associated with symptoms of PTSD are partially attributable to cognitive dysfunction (Geuze, Vermetten, de Kloet, Hijman, \& Westenberg, 2009). Most cognitive research on PTSD symptomatology has focused on learning and memory dysfunctions (Moore, 2009; see Rubin, Berntsen, \& Bohni, 2008, for a review). However, there is emerging evidence that symptoms

Correspondence and reprint requests to: Joseph DeGutis, Geriatric Research Education and Clinical Center (GRECC), Boston VA Healthcare System, Boston, MA 02130. E-mail: degutis@wjh.harvard.edu of PTSD are also associated with poorer performance on executive function tasks (e.g., Esterman et al., 2013a; Polak, Witteveen, Reitsma, \& Olff, 2012; Schuitevoerder et al., 2013; Swick, Honzel, Larsen, Ashley, \& Justus, 2012). Executive functions include task switching, working memory, and inhibitory control that enable complex goal-directed behaviors (Alvarez \& Emory, 2006; Miyake, et al., 2000). Executive functions may be particularly relevant to PTSD symptoms because they are associated with diminished functional status and may compromise PTSD treatments (e.g., emotion regulation strategies during trauma-focused therapies, Mohlman \& Gorman, 2005; Polak, et al., 2012; Vasterling \& Verfaellie, 2009). The current study addresses whether PTSD symptoms are related to general executive dysfunction or are more related to certain executive function subdomains.

Two recent reviews examined the link between PTSD and executive dysfunction. Polak et al. (2012) performed a metaanalysis, examining the results of classic executive functioning tests (e.g., Stroop, Trails B, Digit Span, Wisconsin 
Card Sorting Test) in PTSD compared to trauma-exposed and healthy controls. Supporting a link between PTSD and general executive dysfunction, Polak and colleagues found that patients with PTSD demonstrated significantly poorer performance on Trails B, the Wisconsin Card Sorting Test, and Digit Span than did trauma-exposed controls. Another review (Qureshi et al., 2011) examined a much broader array of cognitive tasks, performed qualitative analyses rather than pooling effect sizes, and found more mixed results. Of the 16 executive function studies reviewed, only 9 found significant deficits in executive function related to PTSD (predominantly on Trails B or Symbol Digit Modalities Test). Contrary to the findings of Polak et al. (2012), Qureshi et al. (2011) did not find a PTSD-related impairment on the Wisconsin Card Sorting Test, likely because these effects were modest and are only significant when using a meta-analytic approach.

One explanation for these mixed findings is that individuals with PTSD have impairments within specific executive functioning subdomains and that measures assessing multiple executive function subdomains simultaneously fail to consistently capture these deficits. In line with this explanation, another review proposed that executive dysfunction associated with symptoms of PTSD is more specific to the subdomain of inhibitory control (Aupperle, Melrose, Stein, \& Paulus, 2012). Inhibitory control involves executive functions related to stopping or changing automatic response patterns (e.g., response inhibition) or control of selective attention (e.g., distractor suppression). Aupperle and colleagues suggest two possible mechanisms relating impaired inhibitory control and PTSD: (1) the re-experiencing and hyperarousal symptoms associated with PTSD interfere with inhibitory control by creating more distractors during daily life; or (2) preexisting inhibitory control dysfunction impairs one's ability to inhibit attending to and disengaging from trauma-related triggers and emotional memories, increasing the likelihood of developing PTSD. Support for the association between inhibitory control and PTSD symptoms comes from studies showing PTSDrelated deficits in distractor suppression (Esterman et al., 2013a; Leskin \& White, 2007) and response inhibition (Swick et al., 2012; Wu et al., 2010). While these studies suggest a prominent relationship between inhibitory control impairments and PTSD symptoms, due to the limited battery of executive function assessments used in these studies, they cannot conclusively determine whether these PTSD-related associations are greater than associations of PTSD with other executive functions.

To better clarify the generality of executive dysfunction in those with PTSD symptoms and test the hypothesis that PTSD symptoms are specifically related to inhibitory control dysfunction, we administered a comprehensive executive functioning battery to a group of OEF/OIF Veterans with varying levels of PTSD symptoms. We included sensitive measures covering the three executive functioning subdomains advocated by Miyake and colleagues (2000)inhibitory control (go/no-go, visual distraction task, Color Word Interference Test-Stroop), working memory (Digit Span, Auditory Consonant Trigrams), and task switching (Intra-Extra Dimensional Set Shift-IED). We also included measures that combined multiple subdomains such as Verbal Fluency (FAS category and letter fluency) and Trails Number Letter Sequencing (Trails NL, analogous to Trails B) from the Delis-Kaplan Executive Function System (DKEFS). Giving such a comprehensive battery to the same group of participants allowed us to test whether participants with greater PTSD symptoms exhibit specifically poorer performance in inhibitory control or more generally poorer performance across all executive functions.

\section{METHODS}

\section{Participants}

Participants were drawn from a pool of $250 \mathrm{OEF} / \mathrm{OIF}$ Veterans recruited into the Traumatic Brain Injury (TBI) Center of Excellence at the Boston VA-the Translational Research Center for TBI and Stress Disorders (TRACTS, for a more indepth description of recruitment and characteristics of this sample, see Amick et al., 2013; Fortier et al., 2013). All participants recruited to TRACTS undergo a comprehensive neuropsychological assessment including standardized tests of executive function, memory, and attention. The final sample of 37 for the present study includes those that were contacted following their TRACTS session to participate in a second session that involved more in-depth assessment of executive functions. [Of the 37 participants in the current study, 17 were previously reported in the study of the Attentional Capture Task by Esterman and colleagues (2013a).] The average time between the TRACTS session and the subsequent executive function session was 5.6 months $(S D=4.6)$. In the TRACTS session, all participants received the Clinician Administered PTSD Scale (Blake et al., 1995) and all had experienced at least one Criterion A traumatic event but only 18 of the 37 had a PTSD diagnosis. As part of the Center's exclusionary criteria, participants did not have a history of neurological/physical impairments, although eight reported mild traumatic brain injury (mTBI). Psychiatric exclusionary criteria included psychotic disorders, bipolar disorder, and suicidal/homicidal ideation requiring intervention. This study was approved by the VA Boston IRB, written consent was obtained from all participants, and research was conducted according to the Declaration of Helsinki.

\section{Paradigms/Procedures}

\section{Clinical measures}

During the TRACTS session, severity of PTSD symptoms was assessed with the PTSD Checklist-Civilian (PCL-C, Weathers, Litz, Herman, Huska, \& Keane, 1994) and the Traumatic Life Experiences Questionnaire (Kubany et al., 2000). The PCL-C is a self-report measure corresponding to the DSM-IV symptoms for PTSD with excellent reliability (full scale, Cronbach's $\alpha=.96$ ). Depressive symptoms were assessed with the Depression Anxiety Stress Scales, Depression subscale (DASS; Lovibond \& Lovibond, 1995; 
Cronbach's $\alpha=.82$ ). To assess trauma exposure and common comorbidities of deployment-related PTSD symptoms, participants completed self-report questionnaires including the Deployment Risk and Resilience Inventory-2 combat experiences portion (DRRI; Vogt, Proctor, King, King, \& Vasterling, 2008), Lifetime Drinking History questionnaire ( $\mathrm{LDH}$, average number of drinks on a drinking day and LDH total corrected for body weight; Skinner \& Sheu, 1982), Short Michigan Alcohol Screening Test (SMAST, total score; Selzer, Vinokur, \& Rooijen, 1975), and the Boston Assessment of Traumatic Brain Injury-Lifetime (BAT-L; Fortier et al., 2013). During the second testing session, participants again completed the PCL-C (first and second session PCL-C correlated $r=.92$ ) and were administered the Beck Depression Inventory-II (BDI-II), which reliably measures depression symptomatology (Cronbach's $\alpha=.92$ ) and correlates highly with the DASS-depression $(\mathrm{r}=.74$; Lovibond \& Lovibond, 1995).

\section{Cognitive measures}

Assessment of executive functions included the following well-validated, reliable measures (see Table 1 for the session during which each measure was given, and see Supplementary Materials for further descriptions).

Task Switching. Participants completed the Intra-Extra Dimensional Set Shift (IED) from the Cambridge Automated Neuropsychological Test Battery (CANTAB, Cambridge, England, 2002), a computer-based analogue to the Wisconsin Card Sorting Test. Dependent measures of interest were the total errors (adjusted score) and the total completed stage trials.

Working Memory. Participants completed the Auditory Consonant Trigrams (ACT) and the Digit Span from the Wechsler Adult Intelligence Scale, Fourth Edition (Wechsler, 1997). The dependent measures were the total number of correct responses for ACT and for Digit span, the sum of the Digit Span Forward, Backward, and Sequencing scores.

Table 1. Measures administered during the first and second testing sessions

First Session Measures

Auditory Consonant Trigrams

Color Word Interference Test (Stroop)

Digit Span

Intra-Extra Dimensional Set Shift

Trails Number Letter Sequencing

Verbal Fluency (category and letter)

Second Session Measures

Capture Task

Gradual Onset Continuous Performance Task
Inhibitory Control. We measured three aspects of inhibitory control: response inhibition, interference control, and distractor suppression. For response inhibition, we used the percent of presses to infrequent no-go stimuli (commission error rate) from a go/no-go continuous performance task, called the gradCPT (gradual onset continuous performance task, Esterman et al., 2014; Rosenberg, Noonan, DeGutis, \& Esterman et al., 2013a; Esterman et al., 2013b). We included the Color Word Interference Test (i.e., Stroop Test) as a measure of inhibition known as "interference control" (Stuss, Floden, Alexander, Levine, \& Katz, 2001). We chose the dependent measure to be interference trial total time instead of difference scores (e.g., the difference between interference trials and color naming or word reading trials) because difference scores are typically less reliable (Peter, Churchill, \& Brown, 1993) and we had no reason to believe that subjects would differ on either color naming or word reading trials. Difference scores were highly correlated with interference trial time ( $\rho$ 's $>.92)$ and all main results replicated when using differences scores. For distractor suppression, participants performed a version of the irrelevant singleton visual search paradigm (i.e., Capture Task, Theeuwes \& Burger, 1998; Esterman et al., 2013a). The dependent measure was the mean reaction time of distractor present trials minus distractor absent trials.

Multiple Executive Subdomains. To examine multiple sub-domains of executive function within single measures, participants also completed the Category and Letter Fluency conditions of the Verbal Fluency Test and the Trail Making Number Letter Sequencing Test (Trails NL, analogous to Trails B) from the Delis-Kaplan Executive Function System (DKEFS, www.pearsonclinical.com, Delis, Kaplan, \& Kramer, 2001). We consider Trails NL to measure "multiple executive subdomains" because studies suggest it engages both working memory and switching (e.g., Sanchez-Cubillo et al., 2009). Additionally, we classify FAS as "multiple executive subdomains" because it requires working memory, inhibition (suppression of irrelevant responses, Burgess \& Shallice, 1996), and engages set shifting mechanisms (Rende, Ramsberger, \& Miyake, 2002). The dependent measure for Trails NL was time to complete whereas the dependent measures for Category and Letter Fluency was total score.

\section{RESULTS}

\section{Participant Characteristics}

Characterization of the 37 participants is shown in Table 2. All were male and 1 participant was African American, 4 were Hispanic, 30 were Caucasian, and 2 participants did not report ethnicity. At the TRACTS baseline assessment 18 of the 37 participants had a PTSD diagnosis based on the Clinician Administered PTSD Scale and endorsed an average of $5.61 \pm 3.08$ lifetime traumatic events on the Traumatic Life Experiences Questionnaire (Kubany et al., 2000), with 30 
Table 2. Characterization of the current OEF/OIF Veteran sample

\begin{tabular}{lcl}
\hline \hline & Mean (SD) & Interpretation \\
\hline Demographics & & \\
$\quad$ Age & 32.8 years $(9.7)$ & \\
Education & 13.9 years $(2.7)$ & \\
Deployment Related Measures & $1.3(0.6)$ & \\
$\quad$ \# of deployments & 12.6 months $(7.8)$ & Moderate exposure \\
Cumulative deployment duration & $14.1(12.1)$ & Moderate PTSD symptoms \\
DRRI (combat exposure) & & Normal range \\
Clinical Measures: Session 1 & $35.2(17.9)$ & Borderline \\
PCL-C total & $6.1(9.4)$ & No problems to borderline \\
DASS Depression & 5.3 drinks $(2.5)$ & \\
LDH-per day & $1391.9 \mathrm{ml} / \mathrm{kg}(1566.6)$ & Moderate PTSD symptoms \\
LDH total-body weight corrected & $2.09(3.5)$ & Mild mood disturbance \\
SMAST total & & $36.9(16.2)$ \\
Clinical Measures: Session 2 & $12.2(10.2)$ & \\
PCL-C total & & \\
BDI-II &
\end{tabular}

Note. DRRI = Deployment Risk and Resilience Inventory; PCL-C = Posttraumatic Stress Disorder Checklist-Civilian version; DASS $=$ Depression Anxiety Stress Scale; LDH $=$ Life-time Drinking History; SMAST $=$ Short Michigan Alcohol Screening Test; BDI-II $=$ Beck Depression Inventory II.

participants reporting trauma that was deployment-related. Of these, seven participants exclusively had military trauma. Only three participants had major depressive disorder according to the structured clinical interview for DSM-IV (SCID). According to the BAT-L, eight participants reported an mTBI during deployment, with all reporting altered mental status. Three participants with a deployment-related mTBI reported loss of consciousness of $0.5,4$, and $7.5 \mathrm{~min}$, suggesting relatively mild mTBIs. Drinking behavior is reported in Table 2 and according to the SCID, no participants reported current use of other substances. However, four reported previous dependence on opioids, six reported previous cannabis dependence, two reported previous cocaine dependence, one reported previous methamphetamine dependence, one reported previous polysubstance dependence, and one reported previous dependence on other substances. In terms of prescription medications, 4 reported taking anti-hypertensives, 1 reported taking diabetes medication, 3 reported taking anti-depressants, 3 reported taking sedative/hypnotics, and 10 reported taking pain medication.

\section{Correlations between Measures of Executive Functioning}

We first assessed how executive function measures correlated with each other. We performed Spearman's $\rho$ correlations rather than Pearson correlations, as some variables were not normally distributed, although all critical results replicate using Pearson correlations. As seen in Figure 1, Trails NL and Verbal Fluency (tasks simultaneously measuring multiple subdomains of executive functioning) were significantly correlated with Digit Span and ACT (tasks specifically measuring working memory), IED errors adjusted

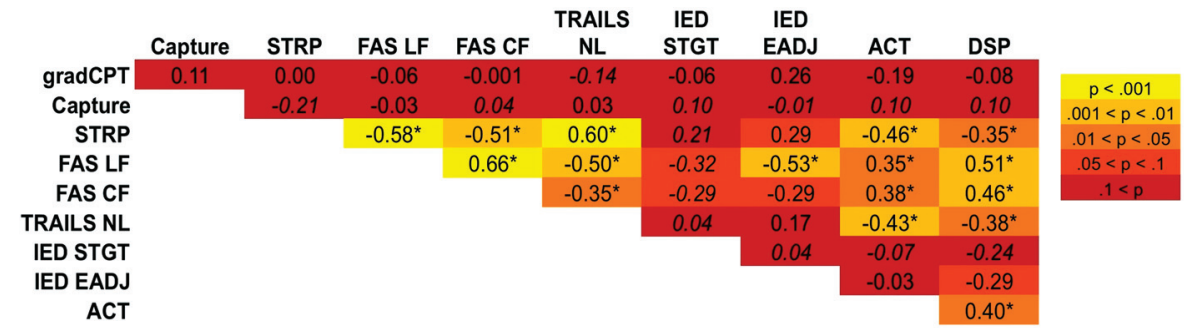

Fig. 1. Spearman correlations between measures of executive functioning. Color indicates strength of correlation. Asterisk indicates $p<.05$. Correlations in the paradoxical direction (enhanced performance on one task correlating with decreased on another) are italicized. As can be seen, measures of response inhibition (gradCPT) and distractor suppression (Capture Task) do not correlate with other executive function tasks or with each other. In contrast, other executive function measures generally correlated with each other and within their subdomains. gradCPT $=$ gradual onset continuous performance task; Capture $=$ Capture Task; STRP $=$ Stroop task; FAS LF $=$ Letter fluency; FAS CF = Category Fluency; Trails NL = Number Letter sequencing; IED STGT $=$ Intra/Extra Dimensional Shift Task stages completed; IED EADJ = Intra/Extra Dimensional Shift Task errors adjusted; ACT $=$ auditory consonant trigrams; DSP $=$ Digit Span. 
(switching), and Stroop, although they were not correlated with the other measures of inhibitory control (gradCPT, Capture Task). Although working memory tasks were correlated with each other, inhibitory control tasks were not correlated with one another. Because in this population the Stroop task significantly correlated with tasks measuring multiple subdomains of executive functioning as well as with working memory tasks, for the regressions below we group Stroop with the "other" executive functions rather than inhibitory control tasks. Note that all key results replicate when grouping Stroop with the inhibitory control tasks (see Supplementary Materials).

\section{Is Inhibitory Control More Strongly Associated with PTSD Symptoms Than Other Executive Functions?}

To test our main hypothesis that PTSD symptoms have a unique association with inhibitory control above and beyond the rest of executive functions, we ran hierarchical regressions comparing inhibitory control measures (gradCPT, Capture Task) to the other executive function measures (FAS LF/CF, Trails NL, IED Stages/Errors, ACT, Digit Span, Stroop) (see Table 3). Since PTSD symptoms measured in the two sessions were very similar and highly correlated, for simplicity we averaged the PCL-C scores from the two sessions to create a composite PCL-C. Results showed only subtle differences when using either the first or second session PCL-C score (see supplementary materials).

We began by entering all executive functions except inhibitory control measures into the model with the composite PCL-C total as the dependent variable. We then entered inhibitory control measures as predictors. The results demonstrate that executive functions without inhibitory control were not associated with a significant amount of variance in PCL-C $\left(\mathrm{R}^{2}=.253\right.$, adjusted $\mathrm{R}^{2}=.039$; $p=.343$ ). Adding inhibitory control measures explained a significant amount of the remaining variance in PCL-C that was not explained by the other executive functions $\left(\mathrm{R}^{2}=.546\right.$, adjusted $\mathrm{R}^{2}=.372, \quad \mathrm{R}^{2}$ change $=.293$; $p=.002)$. These results demonstrate that inhibitory control measures explain significant variance in PTSD symptoms above and beyond the other executive function measures, while other executive measures alone do not explain significant variance in PTSD severity.

We next ran the reverse analyses to determine whether the "other" executive functions are associated with variance in PTSD symptoms above and beyond inhibitory control measures. When inputting inhibitory control measures first and the rest of the executive function measures second, inhibitory control explained significant variance in the composite PCL-C score $\left(\mathrm{R}^{2}=.235\right.$, adjusted $\left.\mathrm{R}^{2}=.190 ; p=.01\right)$. Adding the additional executive functions only showed a trend toward explaining a significant amount of the variance in the PCL-C that was not explained by the inhibitory control measures $\left(\mathrm{R}^{2}=.546\right.$, adjusted $\mathrm{R}^{2}=.372, \mathrm{R}^{2}$ change $\left.=.311 ; p=.058\right)$. This demonstrates that the "other" executive functions do not significantly explain unique variance in PTSD above and beyond inhibitory control. Rerunning these analyses after removing individuals with mTBI showed nearly identical results. We also repeated these analyses on the separate PCL-C subscales and found very similar results, most likely because the subscales were so highly correlated to each other and the overall PCL-C score (all Spearman's $\rho$ 's > .71).

Next, to better characterize the relationship between PTSD symptoms and individual measures, we ran exploratory Spearman correlations (see Figure 2). Only gradCPT and the Capture Task showed an appreciable relationship with PTSD symptoms. It is notable that Stroop interference, although a classic measure of inhibitory control, failed to show a robust relationship with PTSD symptoms. No other executive measures significantly correlated with PCL-C scores. Of

Table 3. Hierarchical regression models predicting PTSD/Depression symptoms from measures of inhibitory control and 'other' executive functions. Significant models are indicated by *.

\begin{tabular}{|c|c|c|c|c|c|c|c|c|c|}
\hline \multirow[b]{3}{*}{ Predictor } & \multicolumn{9}{|c|}{ A. Inhibitory Control Associated with Variance in PTSD/Depression Beyond 'Other' Executive Functions } \\
\hline & \multicolumn{3}{|c|}{$\begin{array}{l}\text { Predicting PTSD (PCL-C Average of } \\
1^{\text {st }} \text { and } 2^{\text {nd }} \text { Session) }\end{array}$} & \multicolumn{3}{|c|}{$\begin{array}{l}\text { Predicting Depression } \\
\text { (DASS } 1^{\text {st }} \text { Session) }\end{array}$} & \multicolumn{3}{|c|}{$\begin{array}{l}\text { Predicting Depression } \\
\text { (BDI-II } 2^{\text {nd }} \text { Session) }\end{array}$} \\
\hline & $\mathrm{R}^{2}$ adj & $\mathrm{R}^{2} / \Delta \mathrm{R}^{2}$ & p-value & $\mathrm{R}^{2}$ adj & $\mathrm{R}^{2} / \Delta \mathrm{R}^{2}$ & p-value & $\mathrm{R}^{2} \mathrm{adj}$ & $\mathrm{R}^{2} / \Delta \mathrm{R}^{2}$ & p-value \\
\hline 1) Switching, Mixed, WM, Stroop & 0.04 & 0.25 & 0.34 & 0.05 & 0.26 & 0.33 & 0.01 & 0.21 & 0.49 \\
\hline \multirow[t]{3}{*}{ 2) Inhibitory Control (except Stroop) } & 0.37 & $0.55 / 0.29$ & $0.002 *$ & 0.18 & $0.41 / 0.15$ & 0.12 & 0.37 & $0.55 / 0.33$ & $0.01 *$ \\
\hline & \multicolumn{9}{|c|}{ B. 'Other' Executive Functions Associated with Variance in PTSD/depression Beyond Inhibitory Control } \\
\hline & \multicolumn{3}{|c|}{$\begin{array}{l}\text { Predicting PTSD (PCL-C Average of } \\
\qquad 1^{\text {st }} \text { and } 2^{\text {nd }} \text { Session) }\end{array}$} & \multicolumn{3}{|c|}{$\begin{array}{l}\text { Predicting Depression } \\
\text { (DASS } 1^{\text {st }} \text { Session) }\end{array}$} & \multicolumn{3}{|c|}{$\begin{array}{l}\text { Predicting Depression } \\
\text { (BDI-II } 2^{\text {nd }} \text { Session) }\end{array}$} \\
\hline Predictor & $\mathrm{R}^{2}$ adj & $\mathrm{R}^{2} / \Delta \mathrm{R}^{2}$ & p-value & $\mathrm{R}^{2} \mathrm{adj}$ & $\mathrm{R}^{2} / \Delta \mathrm{R}^{2}$ & p-value & $\mathrm{R}^{2} \mathrm{adj}$ & $\mathrm{R}^{2} / \Delta \mathrm{R}^{2}$ & $\mathrm{p}$-value \\
\hline 1) Inhibitory Control (except Stroop) & 0.19 & 0.24 & $0.01 *$ & 0.10 & 0.15 & 0.06 & 0.25 & 0.29 & $0.003 *$ \\
\hline 2) Switching, Mixed, WM, Stroop & 0.37 & $0.55 / 0.31$ & 0.06 & 0.18 & $0.41 / 0.25$ & 0.12 & 0.37 & $0.55 / 0.26$ & $0.01 *$ \\
\hline
\end{tabular}




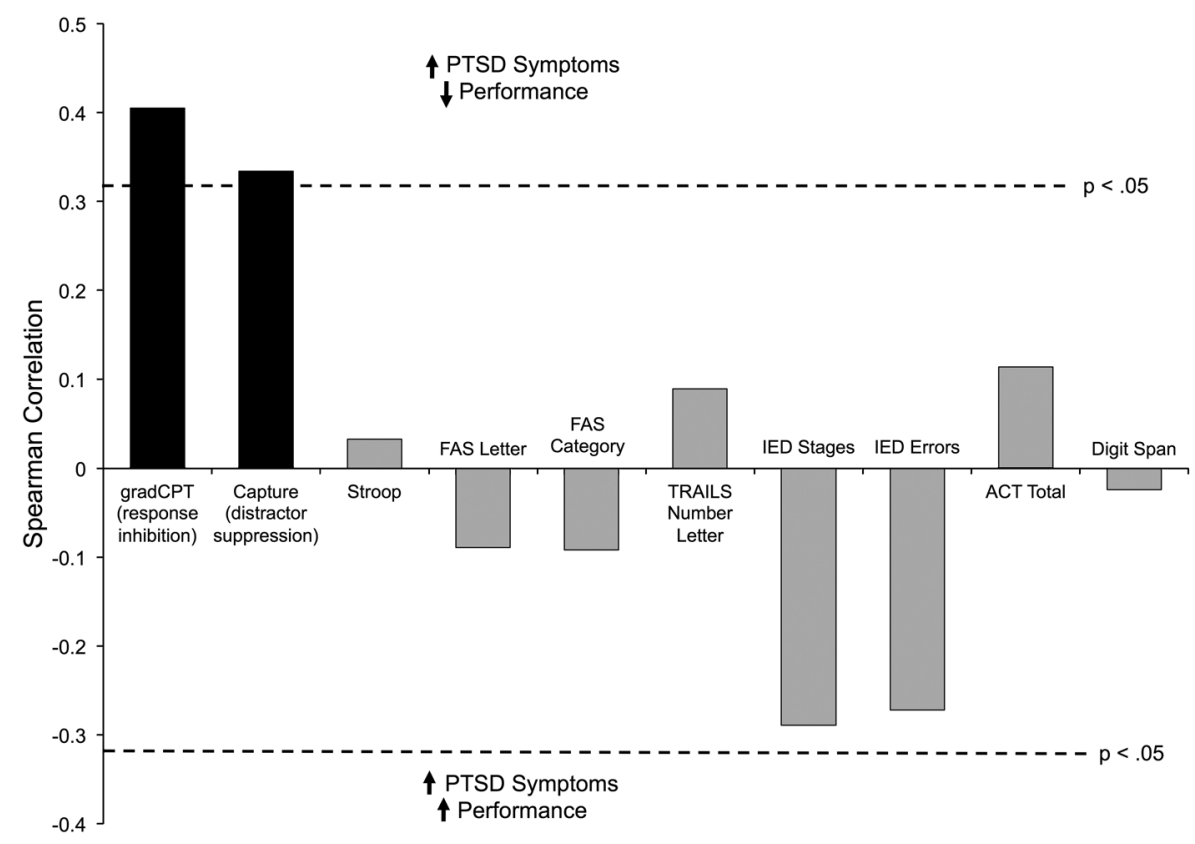

Fig. 2. Spearman correlations between performance on executive functioning tasks and PTSD symptoms (PCL-C). The region above the $\mathrm{X}$-axis indicates the expected direction of the correlation and the dotted lines indicate a significance of $p<.05$ uncorrected for multiple comparisons. Black columns indicate significant correlations. gradCPT $=$ gradual onset continuous performance task; ACT $=$ auditory consonant trigrams. IED = Intra/extradimensional shift task.

interest, the directionality of the correlations between PTSD and task switching (IED completed stage trials and adjusted errors) was not in the predicted direction - those who had fewer errors and completed more stages had more severe PTSD symptoms.

\section{Do the Capture Task and GradCPT Independently Explain Variance in PTSD?}

We next ran exploratory regression analyses to examine whether the Capture Task and gradCPT, the two measures that demonstrated strong associations with PTSD symptoms, are associated with unique variance in PTSD symptoms from each other. We expected that because they were not significantly correlated each, they would be associated with independent variance in PTSD symptoms. As seen in Table 4, the linear regression model showed that the Capture Task and gradCPT explained $26 \%$ of the variance in PTSD symptoms (using the PCL-C given during the second session rather than composite PCL-C), confidence interval [.03, .49]. Notably, the Capture Task and gradCPT were independently associated with variance in PTSD symptoms, suggesting that these tasks explain unique variance in PTSD.

\section{Exploratory Associations between Depression and Executive Functions}

To better understand the nature of the inhibitory control/ PTSD association, we performed exploratory correlations between executive functions and symptoms of depression. Measures of depression performed similarly to PTSD
Table 4. Multiple regression predicting PTSD from measures of inhibitory control

\begin{tabular}{lcc}
\hline \hline & \multicolumn{2}{c}{ Predicting PTSD (PCL-C) } \\
\cline { 2 - 3 } Predictor & $\beta$ & p-value \\
\hline Capture Effect & $0.38^{*}$ & $0.01 *$ \\
Grad CPT Commission Errors & $0.33^{*}$ & $0.03 *$ \\
Adjusted R & \multicolumn{2}{c}{$0.26^{*}$} \\
\hline \hline
\end{tabular}

Note. $\beta=$ standardized beta. ${ }^{*} p<.05$.

measures (these measures are highly correlated: DASS/first session PCL-C, $\rho=.88 ; \mathrm{BDI} /$ second session PCL-C, $\rho=.55)$, with depression strongly correlating with the Capture Task $(\rho=.44 ; p<.05)$ and trending significance with $\operatorname{gradCPT}(\rho=.32 ; p=.05)$. Also, there were no significant correlations between depression and Stroop, Digit Span, ACT, Category Fluency, Letter Fluency, or Trails (all $p$ 's > .22). For IED measures, depression showed a trend toward negatively correlating with IED errors $(\rho=-.29$; $p=.09)$ but not with stages completed $(\rho=.12 ; p=.49)$. Performing hierarchical regressions with depression symptoms showed similar results to PTSD symptoms, particularly when examining the second session BDI scores, with inhibitory control explaining variance above and beyond "other" executive functions (see Table 3). Also paralleling the results with PTSD symptoms, in a linear regression model explaining depression from both the Capture Task and gradCPT, the Capture Task explained independent variance in depression 
(standardized $\beta=.40 ; p<.01$ ) whereas the gradCPT commission errors showed a trend toward this result (standardized $\beta=.30 ; p=.05)$. Together, gradCPT and Capture Task explained $25 \%$ of the variance in depressive symptoms (as compared to $26 \%$ in PTSD symptoms).

\section{Exploratory Associations between Mild TBI/ Drinking/Medications and Executive Functions}

We also explored the relationship between mTBI, drinking, medications and executive functions in this population. In contrast to the results with depression, we found no significant relationship between the presence/absence of a deployment TBI and any of the executive function measures (all p's > .18). This is similar to recent studies of executive function performance in OEF/OIF Veterans by Swick et al. (2012) and Esterman et al. (2013a).

For the drinking variables, we examined $\mathrm{LDH}$ total (corrected for body weight), LDH average drinks on a drinking day, and SMAST total. We used these variables instead of current drinking measures because drinking measures were only given during the first testing session and LDH total, LDH average drinks, and SMAST total are more stable over time than variables such as current drinking amount, making them more valid for both testing sessions. Out of these drinking variables, we did find that greater LDH total significantly correlated with the Capture Task $(\rho=.41$; $p=.01)$ and trended toward a significant association with Digit Span $(\rho=.29 ; p=.08)$ while the other measures were not significant (all $p$ 's $>.10$ ). Importantly, when LDH total and Capture Task were put into a regression predicting PCL-C, both LDH total (standardized $\beta=.33 ; p=.03$ ) and the Capture Task (standardized $\beta=.60 ; p=.001$ ) explained independent variance, thus LDH total could not account for the observed PTSD/Capture Task relationship. Furthermore, including LDH total as an initial predictor in the hierarchical regression analyses (predicting PCL-C composite from inhibitory control and other executive functions) did not significantly affect the results for either model.

For prescription medications, only pain medications had a sufficient number of subjects to perform statistical analyses (10 taking pain medication, 27 not). Independent $t$ tests demonstrated that those on pain medications showed a trend toward performing worse on IED stages $(t(35)=1.88$; $p=.07)$, IED errors $(\mathrm{t}(35)=1.80 ; p=.08)$, and having higher PCL-C scores $(\mathrm{t}(35)=1.90 ; p=.07)$. Despite these trends, including pain medication as an initial predictor in the hierarchical regression analyses (predicting PCL-C composite from inhibitory control and other executive functions) did not significantly affect the results for either model.

\section{DISCUSSION}

The current results demonstrate that, in a cohort of trauma exposed OEF/OIF Veterans, posttraumatic psychological symptoms are uniquely related to inhibitory control dysfunction rather than general executive dysfunction. Moreover, we found that distinct components of inhibitory control-response inhibition and distractor suppressionwere independently associated with stress-related symptoms and together explain $26 \%$ of variance in PTSD and $25 \%$ of variance in depressive symptoms. This re-affirms that trauma-related psychological symptoms are associated with worse cognitive performance in non-emotional contexts and adds to the growing literature suggesting that inhibitory control is an important mechanism in the development and maintenance of posttraumatic symptomatology.

The present findings challenge the view that increased symptoms of PTSD are associated with generally poorer executive function performance. As seen in Figure 2, none of the executive function tasks beyond the gradCPT and Capture Task demonstrated a negative correlation between performance and PTSD symptom severity. In fact, performance on the IED task showed the opposite trend. As these measures are well validated and have previously demonstrated sufficient sensitivity to executive function deficits, these very low or contradictory correlations are not due to poor psychometrics. These results contrast a recent meta-analysis suggesting that those with a PTSD diagnosis demonstrate poorer performance on Trails B, Digit Span, and the Wisconsin Card Sorting Test (Polak et al., 2012). It could be that one needs to have a clear diagnosis of PTSD rather than simply greater PTSD symptoms (as in the current study) to experience executive dysfunction. Although a possibility, the review by Qureshi and colleagues (2011) studied patients with PTSD diagnoses using a broader array of tasks than Polak and colleagues and found that the majority of executive function studies (9/16) failed to demonstrate significant differences between those with PTSD diagnoses and trauma-exposed controls. This review, combined with the current results, suggests caution is warranted when claiming that PTSD and greater PTSD symptoms are associated with general executive dysfunction. One likely possibility is that the effect size of PTSD-related deficits in general executive functioning is quite small and variable across studies and may only manifest when taking a meta-analytic approach.

Rather than general executive dysfunction, the current results emphasize the specific association of inhibitory control dysfunction with posttraumatic psychological symptoms (PTSD and depression). The very similar pattern of results for symptoms of PTSD and depression suggest that the inhibitory control associations reflect common posttraumatic psychological distress rather than symptoms unique to PTSD (e.g., re-experiencing) or depression, and may have common underlying neural substrates. Our distractor-suppression results are consistent with other studies showing that individuals with PTSD have difficulties filtering out both threatrelated distractors (e.g., Fani et al., 2009; Pineles, Shipherd, Welch, \& Yovel, 2007) as well as non-emotional distractors (e.g., Leskin \& White, 2007). Similarly, they are consistent with a study showing increased attentional capture of nonemotional stimuli in depression (Bredemeier et al., 2012). These results suggest that, in those with post-traumatic 
psychological symptoms, attentional failures in the context of threat-related stimuli may represent an exacerbation of a more general deficit in distractor suppression. Our response inhibition results are also consistent with studies showing that PTSD is associated with failure to inhibit pre-potent responses during not-X CPTs (Vasterling \& Verfaellie, 2009) and go/no-go tasks (Swick et al., 2012) as well as with studies showing impaired inhibition during CPT performance in depression (e.g., Porter, Gallagher, Thompson, \& Young, 2003). One potential explanation is that inhibiting excessive arousal-related subcortical/limbic activity associated with posttraumatic psychological symptoms may deplete inhibitory control resources, not allowing for them to be engaged as efficiently for other cognitive operations. This is consistent with results showing that those with more severe PTSD symptoms fail to efficiently engage right-lateralized inhibitory control brain regions in the dorsal and ventral attention networks (Falconer et al., 2008; Jovanovic et al., 2013; Morey, Petty, Cooper, LaBar, \& McCarthy, 2008; Pannu Hayes, LaBar, Petty, McCarthy, \& Morey, 2009). Together, these results support the theory that deficits in inhibitory control are a core aspect of post-traumatic psychological symptoms (Aupperle et al., 2012). By having less of an ability to stop pre-potent impulses, those with greater symptoms of PTSD and depression may both have difficulty establishing control over the focus of their attention and difficulty disengaging from either salient or trauma-related stimuli.

The current study also suggests that distractor suppression and response inhibition may be dissociable in this population and significantly explain independent aspects of posttraumatic symptomatology. Although studies of healthy controls typically find significant associations between distractor suppression and response inhibition (e.g., Friedman \& Miyake, 2004, albeit using slightly different tasks), we found a weak, non-significant correlation between these measures in the current population. Although it is unclear why, one possibility is that the Capture Task predominantly measures inhibitory control over external distractors while the gradCPT predominantly measures inhibitory control over internal distractors (that may interfere more with response control settings) and that these mechanisms are dissociable in the current population. Supporting this idea, the Capture Task has been shown to be relatively more stimulus-driven and less influenced by one's internal goals (Theeuwes, 2010). Furthermore, studies using gradCPT and other go/no-go tasks have found that response control errors are associated with mind wandering and increased activity in the default mode network, a network thought to reflect internal mentation and task-unrelated thoughts (e.g., Christoff, Gordon, Smallwood, Smith, \& Schooler, 2009; Esterman, Noonan, et al. 2013b). Thus, in the current study gradCPT failures may reflect dysfunction of endogenous, or goal-directed attention (mindless responding), while exaggerated attentional capture may reflect dysfunction of exogenous (stimulus-driven) attentional control. This may manifest as the Capture Task and gradCPT reflecting mechanisms specific to particular posttraumatic psychological symptoms. For example, gradCPT dysfunction could be more related to posttraumatic dissociative symptoms, since dissociative symptoms have been linked to mindwandering, lapses of attention, and failure to maintain goaldirected endogenous attentional control (Bruce, Ray, \& Carlson, 2007). On the other hand, attentional capture could be related to hypervigilance, which has been linked to failures to ignore any salient stimuli in the environment, and thus represents dysregulation of exogenous attentional control (Esterman et al., 2013a). Future studies are necessary to tease apart the unique contribution of distractor suppression and response inhibition to posttraumatic symptomatology.

The association between inhibitory control and posttraumatic psychological symptoms could either be due to pretrauma factors such as genetic/temperamental vulnerabilities or may be due to posttrauma factors. It may be that compromised inhibitory control pre-trauma makes it more difficult to inhibit intrusive thoughts or flexibly engage with trauma-related stimuli, leading to more severe posttraumatic stress and depressive symptoms. This risk factor perspective is consistent with findings of Gilbertson and colleagues (2006) who compared cognitive functioning between twins with and without PTSD and found very similar profiles across a variety of cognitive tasks (see also Parslow \& Jorm, 2007). Alternatively, PTSD and depressive symptoms may cause inhibitory control deficits. This may be because exposure to trauma could create salient external distractors (trauma-related stimuli) as well as internal distractors (ruminations, trauma-related thoughts), which overwhelm or preoccupy inhibitory control systems. This is consistent with other theories of generalized anxiety disorders, in which inhibitory control deficits are thought to reflect worry preoccupying cognitive processing associated with inhibitory control (Attentional Control Theory; Eysenck, Derakshan, Santos, \& Calvo, 2007).

Although the results of the current study are promising, they have limitations. First, our sample was relatively small which may have limited our power to detect significant associations between PTSD symptoms and executive functioning measures. In addition, we did not use an exhaustive battery of executive functions and did not include higherlevel executive measures of reasoning, planning, and abstraction (e.g., Tower of London, Raven's Progressive Matrices). Another limitation is that we included only combat-exposed, male Veterans and these Veterans may not be representative of the entire deployed Veteran population. Replicating these results in a civilian population with PTSD symptoms, as well as in females, would test the generality of the current findings. Additionally, because of the high correlation between PTSD and depressive symptoms, we could not determine whether the relationship between inhibitory control and PTSD symptoms is independent of depression. Some studies suggest that depression may be related to impaired inhibitory control (Quinn, Harris, \& Kemp, 2012) and have found impairments on CPT tasks (Porter et al., 2003) and attentional capture tasks (Bredemeier et al., 2012). In fact, some studies have found no effect of PTSD after controlling for depression (Johnsen, Kanagaratnam, \& 
Asbjørnsen, 2008). However, other studies that have covaried the effects of depression find that PTSD-related cognitive impairments are preserved (e.g., Brandes et al., 2002). Future studies with larger samples would be useful to determine whether the observed inhibitory control association is specific to PTSD or instead share common mechanisms with depression. A final limitation of the current study is that it lacks a clearly defined group with PTSD diagnosis, which limits generalizability to clinical settings.

The current study examined multiple aspects of executive functioning in a group of trauma-exposed individuals and revealed that inhibitory control, both of visual distractors and prepotent responses, uniquely predicts posttraumatic psychological symptoms. These findings add support to a recent model asserting the importance inhibitory control in the development and maintenance of trauma-related symptoms (Aupperle et al., 2012). These findings help lay the groundwork for further investigations of the mechanisms of cognitive dysfunction in those with posttraumatic psychological symptoms and may help to develop more effective cognitive treatments (e.g., Kuckertz et al., 2014).

\section{ACKNOWLEDGMENTS}

This research was supported by the Translational Research Center for TBI and Stress Disorders (TRACTS), a VA Rehabilitation Research and Development Traumatic Brain Injury Center of Excellence (B9254-C) and by Veterans Affairs Career Development Award II's given to J.D. and M.E. (VA Clinical Science R\&D Career Development Award to M.E. 1IK2CX000706-01A2).

\section{CONFLICTS OF INTEREST}

None of the authors have conflicts of interest.

\section{Author Contributions}

J.D. developed the study concept. J.D., M.E., W.M., and R.M. contributed to the study design. Testing and data collection were performed by A.R. B.M. performed the data analysis and interpretation under the supervision of J.D. J.D. drafted the paper with contributions from B.M. and M.E. W.M., R.M., and A.R. provided critical revisions.

\section{Supplementary material}

To view Supplementary material for this article, Please vist http://dx.doi.org/10.1017/S1355617715000235

\section{REFERENCES}

Alvarez, J.A., \& Emory, E. (2006). Executive function and the frontal lobes: A meta-analytic review. Neuropsychology Review, 16(1), 17-42. doi:10.1007/s11065-006-9002-x

Amick, M.M., Clark, A., Fortier, C.B., Esterman, M., Rasmusson, A.M., Kenna, A., \& McGlinchey, R. (2013). PTSD modifies performance on a task of affective executive control among deployed $\mathrm{OEF} / \mathrm{OIF}$ veterans with mild traumatic brain injury. Journal of the International Neuropsychological Society, 19(07), 792-801.

Aupperle, R.L., Melrose, A.J., Stein, M.B., \& Paulus, M.P. (2012). Executive function and PTSD: Disengaging from trauma.
Neuropharmacology, 62(2), 686-694. doi:S0028-3908(11) 00078-5 [pii] 10.1016/j.neuropharm.2011.02.008

Blake, D.D., Weathers, F.W., Nagy, L.M., Kaloupek, D.G., Gusman, F.D., Charney, D.S., \& Keane, T.M. (1995). The development of a clinician-administered PTSD scale. Journal of Traumatic Stress, 8, 75-90. doi:10.1002/jts.2490080106

Bliese, P.D., Wright, K.M., Adler, A.B., Cabrera, O., Castro, C.A., \& Hoge, C.W. (2008). Validating the primary care posttraumatic stress disorder screen and the posttraumatic stress disorder checklist with soldiers returning from combat. Journal of Consulting and Clinical Psychology, 76(2), 272-281. doi:200803290-009 [pii] 10.1037/0022-006X.76.2.272

Brandes, D., Ben-Schachar, G., Gilboa, A., Bonne, O., Freedman, S., \& Shalev, A.Y. (2002). PTSD symptoms and cognitive performance in recent trauma survivors. Psychiatry Research, 110(3), 231-238. doi:S0165178102001257 [pii]

Bredemeier, K., Berenbaum, H., Brockmole, J.R., Boot, W.R., Simons, D.J., \& Most, S.B. (2012). A load on my mind: Evidence that anhedonic depression is like multi-tasking. Acta psychologica, 139(1), 137-145. doi:S0001-6918(11)00214-9 [pii] 10.1016/j.actpsy.2011.11.007

Bruce, A.S., Ray, W.J., \& Carlson, R.A. (2007). Understanding cognitive failures: What's dissociation got to do with it? American Journal of Psychology, 120(4), 553-563.

Burgess, P.W., \& Shallice, T. (1996). Response suppression, initiation and strategy use following frontal lobe lesions. Neuropsychologia, 34(4), 263-272. doi:0028-3932(95) 00104-2 [pii]

Christoff, K., Gordon, A.M., Smallwood, J., Smith, R., \& Schooler, J.W. (2009). Experience sampling during fMRI reveals default network and executive system contributions to mind wandering. Proceedings of the National Academy if Sciences of the United States of America, 106(21), 8719-8724. doi:0900234106 [pii] 10.1073/pnas.0900234106

Delis, D.C., Kaplan, E., \& Kramer, J.H. (2001a). Delis-Kaplan Executive Function System (D-KEFS). San Antonio, TX: The Psychological Corporation.

Esterman, M., DeGutis, J., Mercado, R., Rosenblatt, A., Vasterling, J.J., Milberg, W., \& McGlinchey, R. (2013a). Stress-related psychological symptoms are associated with increased attentional capture by visually salient distractors. Journal of the International Neuropsychological Society, 19(7), 835-840. doi:S135561771300057X [pii] 10.1017/S135561771300057X

Esterman, M., Noonan, S.K., Rosenberg, M., \& Degutis, J. (2013b). In the zone or zoning out? Tracking behavioral and neural fluctuations during sustained attention. Cerebral Cortex, 23(11), 2712-2723.

Esterman, M., Rosenberg, M.D., \& Noonan, S.K. (2014). Intrinsic fluctuations in sustained attention and distractor processing. The Journal of Neuroscience, 34(5), 1724-1730.

Eysenck, M.W., Derakshan, N., Santos, R., \& Calvo, M.G. (2007). Anxiety and cognitive performance: Attentional control theory. Emotion, 7(2), 336-353. doi:2007-06782-011 [pii] 10.1037/ 1528-3542.7.2.336

Falconer, E., Bryant, R., Felmingham, K.L., Kemp, A.H., Gordon, E., Peduto, A., ... Williams, L.M. (2008). The neural networks of inhibitory control in posttraumatic stress disorder. Journal of Psychiatry \& Neuroscience, 33(5), 413-422.

Fani, N., Kitayama, N., Ashraf, A., Reed, L., Afzal, N., Jawed, F., \& Bremner, J.D. (2009). Neuropsychological functioning in patients with posttraumatic stress disorder following short-term paroxetine treatment. Psychopharmacology Bulletin, 42(1), 53-68. 
Fortier, C.B., Amick, M.A., Grande, L., McGlynn, S., Kenna, A., Morra, L., .. McGlinchey, R.E. (2013). The Boston Assessment of Traumatic Brain Injury-Lifetime (BAT-L) semi-structured interview: Preliminary evidence of research utility and validity. The Journal of Head Trauma. [Epub ahead of print]. doi:10.1097/ HTR.0b013e3182865859

Friedman, N.P., \& Miyake, A. (2004). The relations among inhibition and interference control functions: A latent-variable analysis. Journal of Experimental Psychology: General, 133(1), 101-135. doi:10.1037/0096-3445.133.1.1012004-10964-007 [pii]

Geuze, E., Vermetten, E., de Kloet, C.S., Hijman, R., \& Westenberg, H.G. (2009). Neuropsychological performance is related to current social and occupational functioning in veterans with posttraumatic stress disorder. Depression \& Anxiety, 26(1), 7-15. doi:10.1002/da.20476

Gilbertson, M.W., Paulus, L.A., Williston, S.K., Gurvits, T.V., Lasko, N.B., Pitman, R.K., \& Orr, S.P. (2006). Neurocognitive function in monozygotic twins discordant for combat exposure: Relationship to posttraumatic stress disorder. Journal of Abnormal Psychology, 115(3), 484-495. doi:2006-09167-010 [pii] 10.1037/0021-843X.115.3.484

Johnsen, G.E., Kanagaratnam, P., \& Asbjørnsen, A.E. (2008). Memory impairments in posttraumatic stress disorder are related to depression. Journal of Anxiety Disorders, 22(3), 464-474. doi: S0887-6185(07)00104-1 [pii] 10.1016/j.janxdis.2007.04.007

Jovanovic, T., Ely, T., Fani, N., Glover, E.M., Gutman, D., Tone, E.B., ... Ressler, K.J. (2013). Reduced neural activation during an inhibition task is associated with impaired fear inhibition in a traumatized civilian sample. Cortex, 49(7), 1884-1891. doi: S0010-9452(12)00252-3 [pii] 10.1016/j.cortex.2012.08.011

Kessler, R.C., Berglund, P., Demler, O., Jin, R., Merikangas, K.R., \& Walters, E.E. (2005). Lifetime prevalence and age-of-onset distributions of DSM-IV disorders in the National Comorbidity Survey Replication. Archives of General Psychiatry, 62(6), 593602. doi:62/6/593 [pii] 10.1001/archpsyc.62.6.593

Kubany, E.S., Haynes, S.N., Leisen, M.B., Owens, J.A., Kaplan, A.S., Watson, S.B., \& Burns, K. (2000). Development and preliminary validation of a brief broad-spectrum measure of trauma exposure: The Traumatic Life Events Questionnaire. Psychological Assessment, 12, 210-224.

Kuckertz, J.M., Amir, N., Boffa, J.W., Warren, C.K., Rindt, S.E., Norman, S., ... McLay, R. (2014). The effectiveness of an attention bias modification program as an adjunctive treatment for post-traumatic stress disorder. Behaviour Research and Therapy, 63, 25-35.

Leskin, L.P., \& White, P.M. (2007). Attentional networks reveal executive function deficits in posttraumatic stress disorder. Neuropsychology, 21(3), 275-284. doi:2007-06185-001 [pii] 10.1037/0894-4105.21.3.275

Lovibond, P.F., \& Lovibond, S.H. (1995). The structure of negative emotional states: comparison of the Depression Anxiety Stress Scales (DASS) with the Beck Depression and Anxiety Inventories. Behaviour Research \& Therapy, 33(3), 335-343. doi:0005-7967(94)00075-U [pii]

Miyake, A., Friedman, N.P., Emerson, M.J., Witzki, A.H., Howerter, A., \& Wager, T.D. (2000). The unity and diversity of executive functions and their contributions to complex "Frontal Lobe" tasks: A latent variable analysis. Cognitive Psychology, 41(1), 49-100. doi:10.1006/cogp.1999.0734S0010-0285(99) 90734-X [pii]

Mohlman, J., \& Gorman, J.M. (2005). The role of executive functioning in CBT: A pilot study with anxious older adults.
Behaviour Research \& Therapy, 43(4), 447-465. doi:S00057967(04)00086-5 [pii] 10.1016/j.brat.2004.03.007

Moore, S.A. (2009). Cognitive abnormalities in posttraumatic stress disorder. Currernt Opinion in Psychiatry, 22(1), 19-24. doi:10.1097/YCO.0b013e328314e3bb00001504-200901 000-00005 [pii]

Morey, R.A., Petty, C.M., Cooper, D.A., LaBar, K.S., \& McCarthy, G. (2008). Neural systems for executive and emotional processing are modulated by symptoms of posttraumatic stress disorder in Iraq War veterans. Psychiatry Research: Neuroimaging, 162(1), 59-72. doi:S0925-4927(07)00151-5 [pii] 10.1016/j.pscychresns. 2007.07.007

Müller, H.J., Geyer, T., Zehetleitner, M., \& Krummenacher, J. (2009). Attentional capture by salient color singleton distractors is modulated by top-down dimensional set. Journal of Experimental Psychology: Human Perception and Performance, 35(1), 1. doi:2009-00768-001 [pii] 10.1037/0096-1523.35.1

Pannu Hayes, J., LaBar, K.S., Petty, C.M., McCarthy, G., \& Morey, R.A. (2009). Alterations in the neural circuitry for emotion and attention associated with posttraumatic stress symptomatology. Psychiatry Research: Neuroimaging, 172(1), 7-15. doi:S09254927(08)00085-1 [pii] 10.1016/j.pscychresns.2008.05.005

Parslow, R.A., \& Jorm, A.F. (2007). Pretrauma and posttrauma neurocognitive functioning and PTSD symptoms in a community sample of young adults. American Journal of Psychiatry, 164(3), 509-515. doi:164/3/509 [pii] 10.1176/appi.ajp.164.3.509

Peter, J.P., Churchill, G.A. Jr., \& Brown, T.J. (1993). Caution in the use of difference scores in consumer research. Journal of Consumer Research, 655-662.

Pineles, S.L., Shipherd, J.C., Welch, L.P., \& Yovel, I. (2007). The role of attentional biases in PTSD: Is it interference or facilitation? Behaviour Research \& Therapy, 45(8), 1903-1913. doi:S00057967(06)00204-X [pii] 10.1016/j.brat.2006.08.021

Polak, A.R., Witteveen, A.B., Reitsma, J.B., \& Olff, M. (2012). The role of executive function in posttraumatic stress disorder: A systematic review. Journal of Affective Disorders, 141(1), 11-21. doi:S0165-0327(12)00007-9 [pii] 10.1016/j.jad.2012. 01.001

Porter, R.J., Gallagher, P., Thompson, J.M., \& Young, A.H. (2003). Neurocognitive impairment in drug-free patients with major depressive disorder. The British Journal of Psychiatry, 182(3), 214-220.

Quinn, C.R., Harris, A., \& Kemp, A.H. (2012). The impact of depression heterogeneity on inhibitory control. Austrailia New Zealand Journal of Psychiatry, 46(4), 374-383. doi:00048674 11432073 [pii] 10.1177/0004867411432073

Qureshi, S.U., Long, M.E., Bradshaw, M.R., Pyne, J.M., Magruder, K.M., Kimbrell, T., ... Kunik, M.E. (2011). Does PTSD impair cognition beyond the effect of trauma? Journal of Neuropsychiatry \& Clinical Neuroscience, 23, 16-28. doi:23/1/ 16 [pii] 10.1176/appi.neuropsych.23.1.16

Rende, B., Ramsberger, G., \& Miyake, A. (2002). Commonalities and differences in the working memory components underlying letter and category fluency tasks: A dual-task investigation. Neuropsychology, 16(3), 309.

Rosenberg, M., Noonan, S., DeGutis, J., \& Esterman, M. (2013). Sustaining visual attention in the face of distraction: A novel gradual-onset continuous performance task. Attention, Perception, \& Psychophysics, 75(3), 426-439. doi:10.3758/s13414-0120413-x

Rubin, D.C., Berntsen, D., \& Bohni, M.K. (2008). A memory-based model of posttraumatic stress disorder: Evaluating basic 
assumptions underlying the PTSD diagnosis. Psychological Reviews, 115 (4), 985-1011. doi:2008-14936-017 [pii] 10.1037/ a0013397

Sanchez-Cubillo, I., Perianez, J.A., Adrover-Roig, D., RodriguezSanchez, J.M., Rios-Lago, M., Tirapu, J., ... Barcelo, F. (2009). Construct validity of the Trail Making Test: Role of taskswitching, working memory, inhibition/interference control, and visuomotor abilities. Journal of the International Neuropsychological Society, 15(03), 438-450. doi:S1355617709090626 [pii] $10.1017 / \mathrm{S} 1355617709090626$

Schuitevoerder, S., Rosen, J.W., Twamley, E.W., Ayers, C.R., Sones, H., Lohr, J.B., ... Thorp, S.R. (2013). A meta-analysis of cognitive functioning in older adults with PTSD. Journal of Anxiety Disorders, 27(6), 550-558. doi:S0887-6185(13)00003-0 [pii] 10.1016/j.janxdis.2013.01.001

Selzer, M.L., Vinokur, A., \& Rooijen, L.V. (1975). A selfadministered short Michigan alcoholism screening test (SMAST). Journal of Studies on Alcohol and Drugs, 36(01), 117.

Skinner, H.A., \& Sheu, W.J. (1982). Reliability of alcohol use indices; the lifetime drinking history and the MAST. Journal of Studies on Alcohol and Drugs, 43(11), 1157.

Stuss, D.T., Floden, D., Alexander, M.P., Levine, B., \& Katz, D. (2001). Stroop performance in focal lesion patients: Dissociation of processes and frontal lobe lesion location. Neuropsychologia, 39(8), 771-786. doi:S0028-3932(01) 00013-6 [pii]

Swick, D., Honzel, N., Larsen, J., Ashley, V., \& Justus, T. (2012). Impaired response inhibition in veterans with post-traumatic stress disorder and mild traumatic brain injury. Journal of the International Neuropsychological Society, 18(5), 917-926. doi: S1355617712000458 [pii] 10.1017/S1355617712000458
Tanielian, T., \& Jaycox, L. (eds.) 2008). Invisible wounds of war: Psychological and cognitive injuries, their consequences, and services to assist recovery. Santa Monica, CA: RAND Corporation. doi:10.1093/occmed/kqp006

Theeuwes, J., \& Burger, R. (1998). Attentional control during visual search: The effect of irrelevant singletons. Journal of Experimental Psychology: Human Perception and Performance, 24(5), 1342-1353.

Theeuwes, J. (2010). Top-down and bottom-up control of visual selection. Acta Psychologica, 135(2), 77-99. doi:S0001-6918 (10)00042-9 [pii] 10.1016/j.actpsy.2010.02.006

Vasterling, J.J., \& Verfaellie, M. (2009). Introduction-posttraumatic stress disorder: A neurocognitive perspective. Journal of the International Neuropsychological Society, 15(6), 826-829. doi: S1355617709990683 [pii] 10.1017/S1355617709990683

Vogt, D.S., Proctor, S.P., King, D.W., King, L.A., \& Vasterling, J.J. (2008). Validation of scales from the Deployment Risk and Resilience Inventory in a sample of Operation Iraqi Freedom veterans. Assessment, 15(4), 391-403. doi:1073191108316030 [pii] 10.1177/1073191108316030

Weathers, F.W., Litz, B.T., Herman, D., Huska, J., \& Keane, T. (1994). The PTSD checklist-civilian version (PCL-C). Boston, MA: National Center for PTSD.

Wechsler, D. (1997). Manual for the Wechsler Adult Intelligence Scale-III. San Antonio, TX: Psychological Corporation.

Wu, J., Ge, Y., Shi, Z., Duan, X., Wang, L., Sun, X., ... Zhang, K. (2010). Response inhibition in adolescent earthquake survivors with and without posttraumatic stress disorder: A combined behavioral and ERP study. Neuroscience Letters, 486(3), 117-121. doi:S0304-3940(10)00951-1 [pii] 10.1016/j.neulet. 2010.07.040 